\title{
Dual role of nNOS in ischemic injury and preconditioning
}

Anupama Barua', Nicholas B Standen², Manuel Galiñanes ${ }^{1,3^{*}}$

\begin{abstract}
Background: Nitric oxide (NO) is cardioprotective and a mediator of ischemic preconditioning (IP). Endothelial nitric oxide synthase (eNOS) is protective against myocardial ischemic injury and a component of IP but the role and location of neuronal nitric oxide synthase (nNOS) remains unclear. Therefore, the aims of these studies were to: (i) investigate the role of nNOS in ischemia/reoxygenation-induced injury and IP, (ii) determine whether its effect is species-dependent, and (iii) elucidate the relationship of nNOS with mitoK ATP channels and p38MAPK, two key components of IP transduction pathway.

Results: Ventricular myocardial slices from rats and wild and nNOS knockout mice, and right atrial myocardial slices from human were subjected to $90 \mathrm{~min}$ ischemia and $120 \mathrm{~min}$ reoxygenation $\left(37^{\circ} \mathrm{C}\right)$. Specimens were randomized to receive various treatments ( $n=6 /$ group). Both the provision of exogenous $N O$ and the inhibition of endogenous NO production significantly reduced tissue injury (creatine kinase release, cell necrosis and apoptosis), an effect that was species-independent. The cardioprotection seen with nNOS inhibition was as potent as that of $I P$, however, in nNOS knockout mice the cardioprotective effect of non-selective NOS (L-NAME) and selective nNOS inhibition and also that of IP was blocked while the benefit of exogenous NO remained intact. Additional studies revealed that the cardioprotection afforded by exogenous $\mathrm{NO}$ and by inhibition of nNOS were unaffected by the mitoK $_{\text {ATP }}$ channel blocker 5-HD, although it was abrogated by p38MAPK blocker SB203580.
\end{abstract}

Conclusions: nNOS plays a dual role in ischemia/reoxygenation in that its presence is necessary to afford cardioprotection by IP and its inhibition reduces myocardial ischemic injury. The role of nNOS is species-

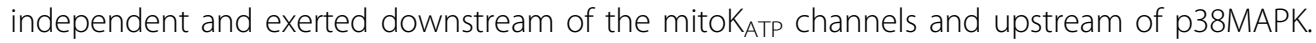

\section{Background}

It is well established that enhanced bioavailability of endogenous nitric oxide (NO) affords cardioprotection against ischemia and reoxygenation-induced injury [1]. This important mediator is enzymatically produced by the NO-synthases (NOSs). There are three NOS isoforms, widely distributed through most cells and tissues, which can produce $\mathrm{NO}$ by converting L-arginine to $\mathrm{L}$-citruline in the presence of $\mathrm{NADPH}, \mathrm{O}_{2}$ and other cofactors [2]. The endothelial NOS (eNOS) and neuronal NOS (nNOS) are constitutive and $\mathrm{Ca}++$-dependent whereas the expression of the inducible NOS (iNOS) is $\mathrm{Ca}++-$ independent and stress-induced.

\footnotetext{
* Correspondence: manuel.galinanes@gmail.com

'Cardiac Surgery Unit, Dept. of Cardiovascular Sciences, University of Leicester, UK

Full list of author information is available at the end of the article
}

It has been documented that nNOS is located in the peripheral vagal nerves, sympathetic nerves and in the autonomic control region of the central nervous system [2]. In cardiomyocytes, nNOS is expressed in sarcoplasmic reticulum, sarcolemma and mitochondria [3-5]. NO derived from nNOS influences the beat to beat regulation of basal cardiac function, serving negative feedback control of $\mathrm{Ca}^{++}$fluxes [6,7]. In addition, it facilitates vagal stimulation and inhibits sympathetic stimulation by exerting its effect in the central and peripheral nervous system.

NO derived from eNOS is essential for eliciting early ischemic preconditioning (IP) [8], however, there is still controversy about the role of nNOS. Thus, although nNOS expression is increased in the acute phase of myocardial infarction in both infarcted and noninfarcted tissues in the rat heart [9], it has been reported that in the nNOS knockout mice, there is no change in 
myocardial infarct size $[10,11]$. By contrast, it appears that delayed IP (72 hours) is dependent on nNOS in the rabbit [12], whereas the deficit of nNOS reduces cerebral ischemic injury in mice [13].

MitoK $_{\text {ATP }}$ channels, located in the inner mitochondrial membrane, are central for IP [14]. Few studies have shown a link between mitoK $\mathrm{ATP}_{\mathrm{T}}$ and NO in IP. Sasaki et al suggested that $\mathrm{NO}$ causes the opening of mitoK $\mathrm{K}_{\mathrm{ATP}}$ directly and indirectly [15] which in turn induces blunting of the $\mathrm{Ca}^{++}$overload in mitochondria, thus contributing to the delayed IP. It has also been reported that the opening of the mitoK $\mathrm{K}_{\mathrm{ATP}}$ channels by diazoxide induces cardioprotection in a NO-dependent manner [16]. Furthermore, Rakhit et al have demonstrated that the exogenous NO donor SNAP induces early IP by a cGMP-dependent mechanism and delayed IP by a cGMP-independent mechanism which is mediated by activation of PKC and mitoK $\mathrm{ATP}_{\mathrm{T}}$ channels [17]. Interestingly, Nakano et al suggested that SNAP-induced cardioprotection cannot be reproduced by endogenous NO [18] also implying that there are two separate pathways for NO-induced cardioprotection.

Our laboratory has previously demonstrated that opening of p38MAPK is an obligatory step in IP that lies downstream of PKC activation [19]. However, there is little information regarding the relationship of NO with p38MAPK in the myocardium. Thus, for example, Kim et al. showed that sodium nitroprusside activates p42/44 and p38MAPK in adult rat cardiomyocytes via both cGMP-dependent and cGMP-independent mechanisms [20]. In another study, Wang et al demonstrated that NO suppressed the angiotensin-I induced activation of ERK in cardiac fibroblast [21]. Other studies carried out in non-cardiac mitogenic cells have shown that NO induced apoptosis is mediated directly by activation of MAPK [20-22]. So, the relationship of NO with p38MAPK remains largely undefined.

Therefore, the aims of the present studies were to: (i) elucidate the role of nNOS in ischemia/reoxygenationinduced injury and IP, (ii) determine whether its effect is species-dependent, and (iii) investigate the relationship of nNOS with mitoK ATP $_{\text {ATannels and }}$ p38MAPK, two key components of the intracellular transduction pathway of protection by IP.

\section{Results}

Myocardial viability, as assessed by the absence of necrosis and apoptosis by the end of the experimental period in aerobically incubated myocardial slices, was greater than $95 \%$ in all instances.

\section{(i) The role of nNOS in ischemic injury}

Figures 1a-c show that, in the rat myocardium, the selective inhibition of nNOS by TRIM resulted in a
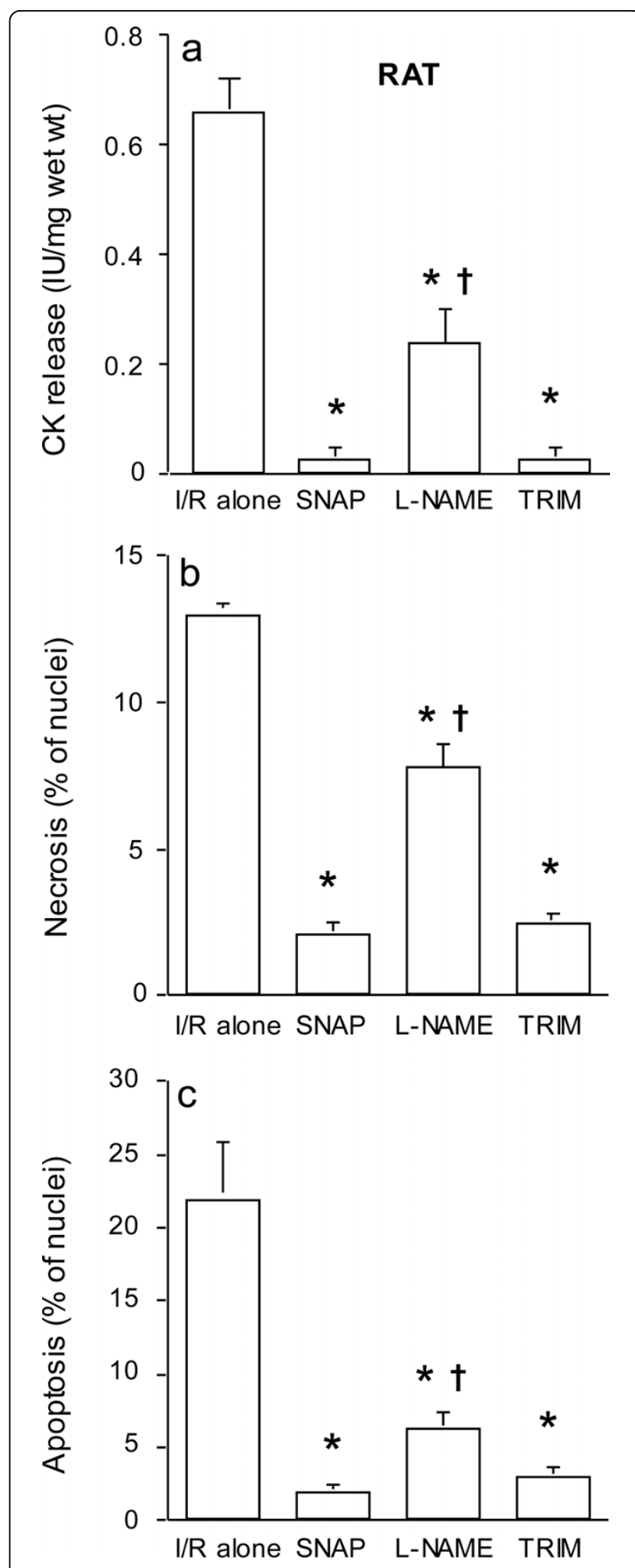

Figure $1 \mathrm{CK}$ release (a), and cell necrosis (b) and apoptosis (c) in rat ventricular myocardium subjected to $90 \mathrm{~min}$ ischemia followed by $120 \mathrm{~min}$ of reoxygenation ( $n=6 /$ group).

Specimens were incubated with SNAP, L-NAME or TRIM for $20 \mathrm{~min}$ prior to ischemia. ${ }^{*} P<0.05$ vs. ischemia/reoxygenation (I/R) alone; †P $<0.05$ vs. SNAP and TRIM treated groups. 
significant reduction in CK release and in cell necrosis and apoptosis, as compared to the mean values in the ischemia/reoxygenation alone group. The degree of this protection was similar to administration of exogenous NO but greater than the non-selective NOS inhibitor LNAME.

\section{(ii) Is the role of nNOS species-dependent?}

As seen in Figures 2a-f, and in comparison with the results observed in Figures 1a-c, the order of magnitude of ischemic injury for CK release and cell necrosis and apoptosis varied slightly between species, however, identical effects on NOS inhibition and from the administration of exogenous NO to that seen in rat myocardium (Figures 1a-c) were observed for both mouse and human myocardium.

\section{(iii) Knocking out nNOS}

Figures 3a-c demonstrate that, as opposed to wild type mice, the myocardium of nNOS knockout mice could not be protected by the specific NOS inhibitor TRIM as the mean values for $\mathrm{CK}$ release and cell necrosis and
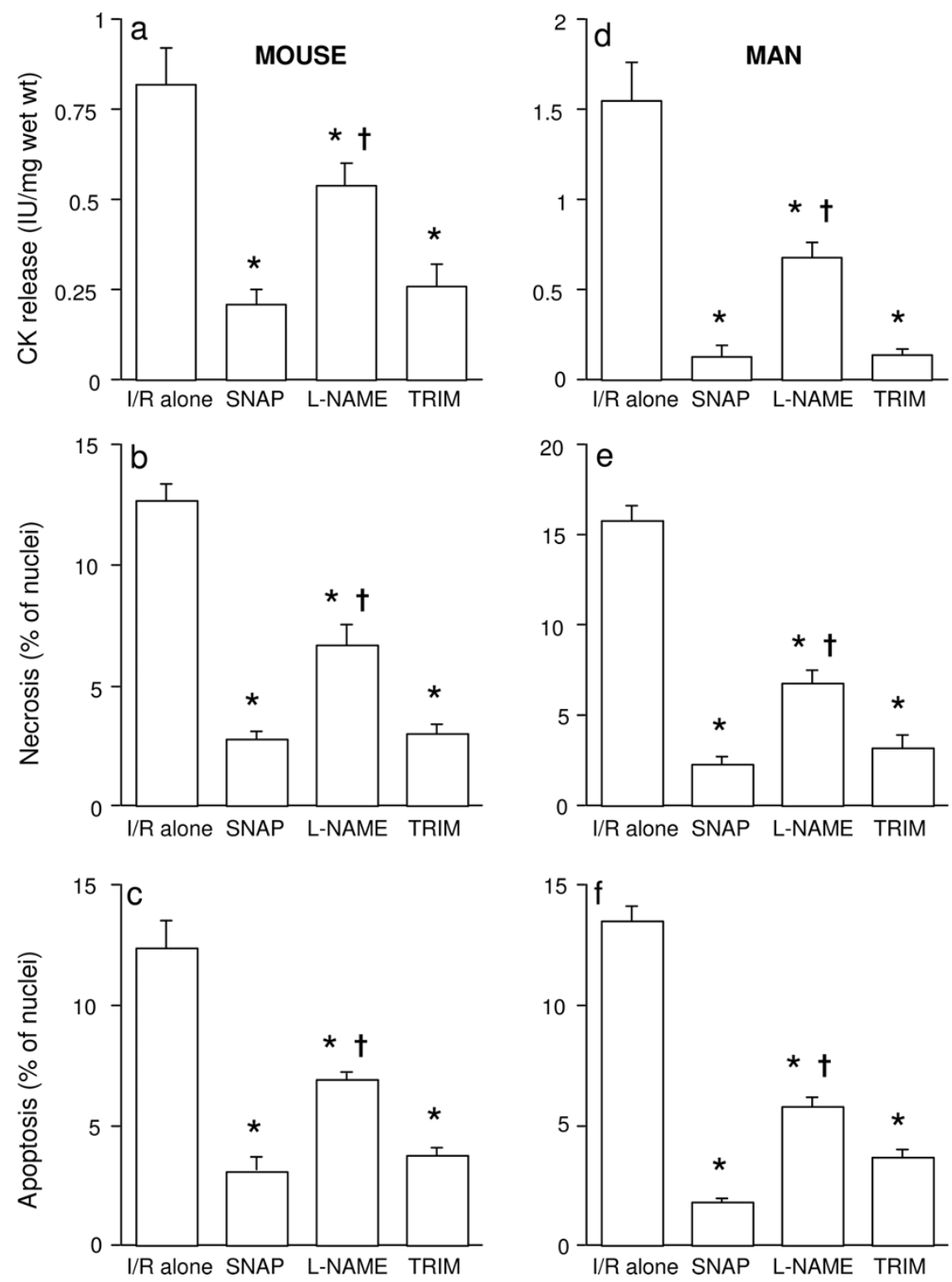

Figure 2 CK release (a), and cell necrosis (b) and apoptosis (c) in mouse ventricular myocardium and CK release (d), and cell necrosis (e) and apoptosis (f) in human right atrial myocardium subjected to 90 min ischemia followed by 120 min of reoxygenation ( $\mathrm{n}=6 /$ group). Specimens were incubated with SNAP, L-NAME or TRIM for 20 min prior to ischemia. ${ }^{*} P<0.05$ vs. ischemia/reoxygenation (I/R) alone; tP $<0.05$ vs. SNAP and TRIM treated groups. 


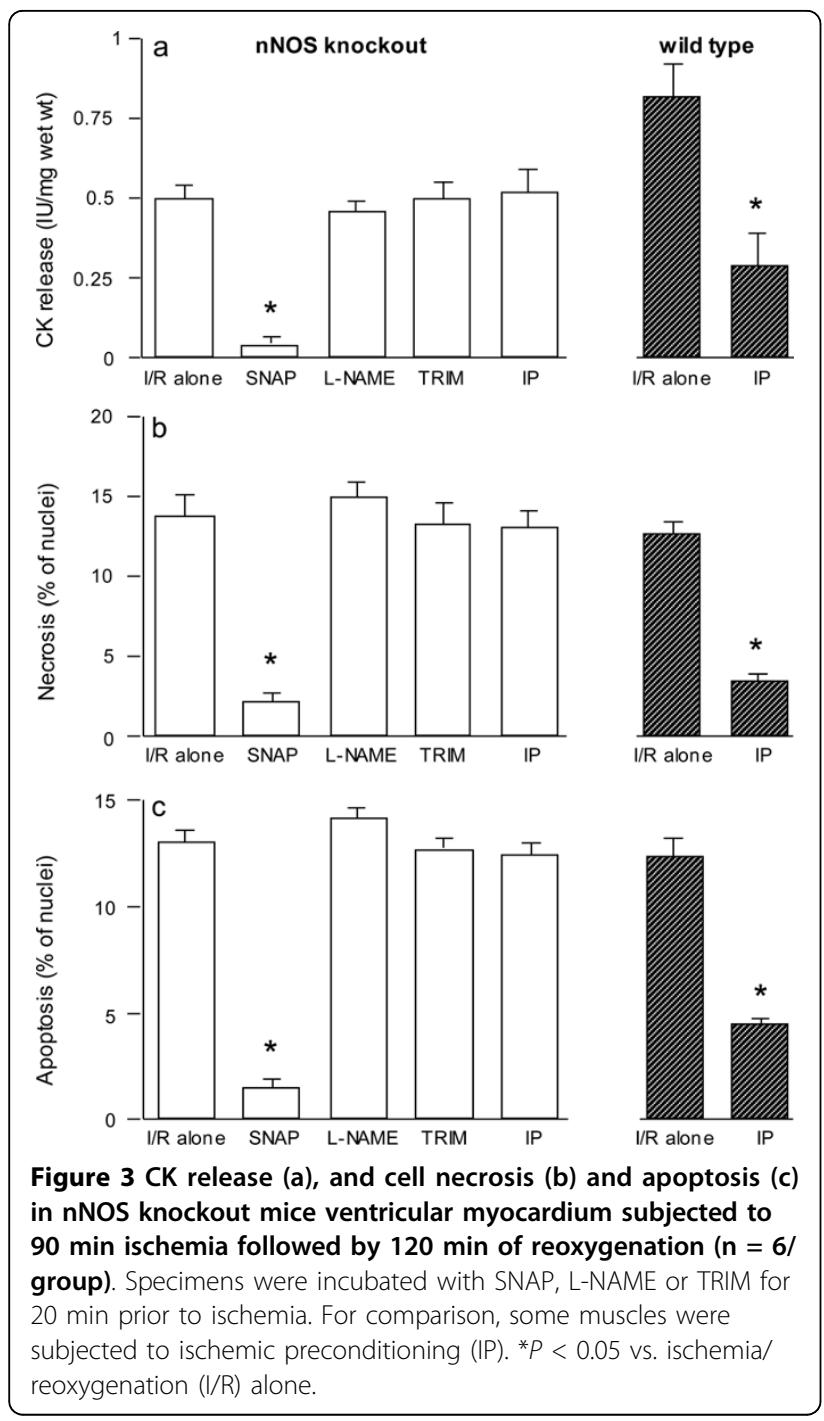

apoptosis were not significantly different from muscles subjected to ischemia/reoxygenation alone. The absence of benefit when L-NAME was added to the myocardium of knockout nNOS mice supports the thesis that although the endogenously produced NO by eNOS may be beneficial its deficit did not cause further ischemic injury. As seen above, again the NO donor SNAP almost abolished the CK release and the cell death induced by ischemia and reoxygenation. Importantly, and in contrast with wild type mice, the myocardium of nNOS knockout out mice could not be protected by IP since the mean values for CK release and cell necrosis and apoptosis did not differ from the values in the ischemia/ reoxygenation alone group. However, the degree of injury in the ischemia-reoxygenation alone groups was similar in both wild type and nNOS knockout mice as previously reported [10]. (iv) The relationship of exogenous NO and $\mathrm{nNOS}$ inhibition with mitoK $\mathrm{K}_{\mathrm{ATP}}$ channels and $\mathrm{p} 38 \mathrm{MAPK}$

Figures $4 \mathrm{a}-\mathrm{c}$ demonstrate that the reduction in CK release and in cell necrosis and apoptosis caused by the NO donor SNAP and the inhibition of nNOS with TRIM, was unaffected by the mitoK $\mathrm{ATP}_{\mathrm{AT}}$ channel blocker 5-HD, and that, as expected, it blocked the protection by IP. By contrast, Figures $5 \mathrm{a}-\mathrm{c}$ show that the p38MAPK blocker SB203580 abolished the protective effect of exogenous NO and of nNOS inhibition, along with the benefit of IP.

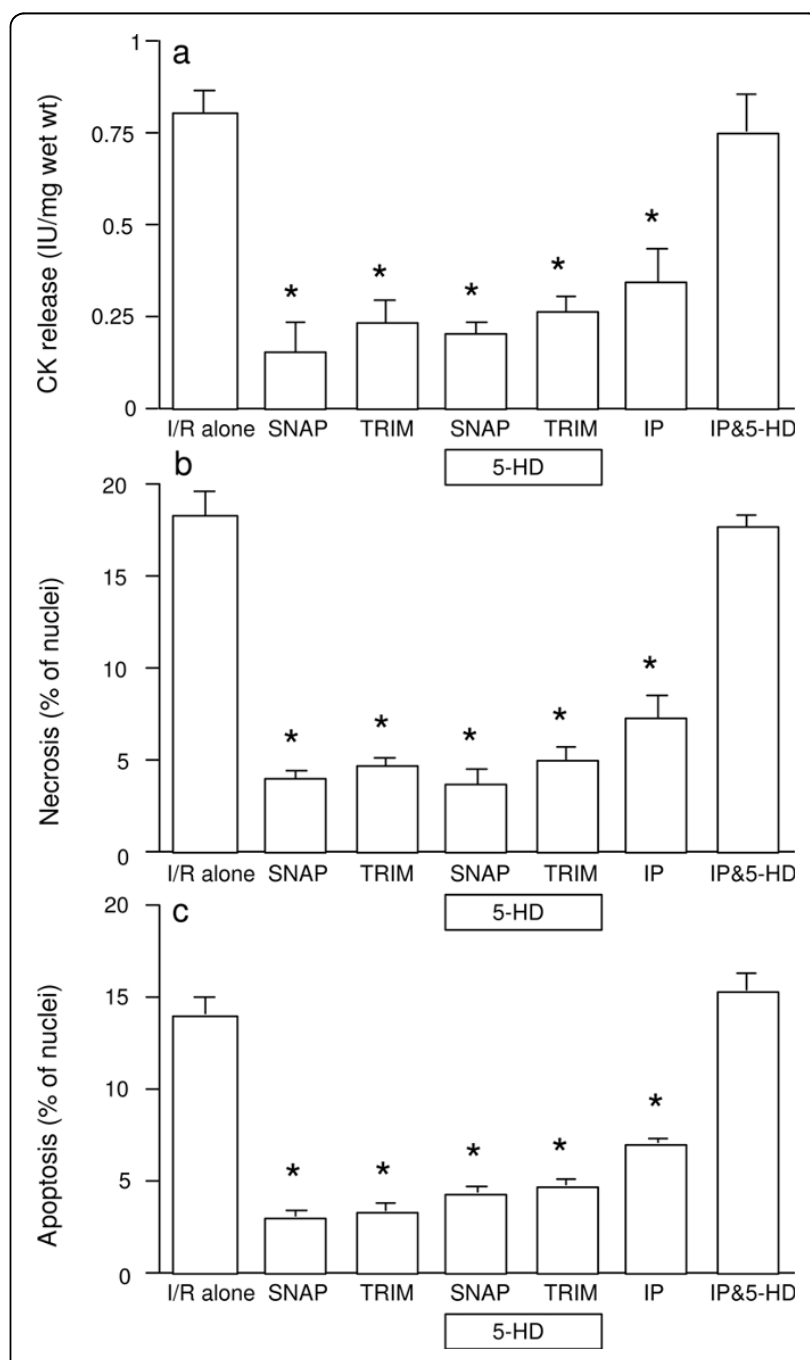

Figure 4 CK release (a), and cell necrosis (b) and apoptosis (c) in rat ventricular myocardium subjected to $90 \mathrm{~min}$ ischemia followed by $120 \mathrm{~min}$ of reoxygenation ( $n=6 /$ group).

Specimens were incubated with SNAP or TRIM for 20 min prior to ischemia in the absences and presence of 5-HD. For comparisons, some muscles were subjected to ischemic preconditioning (IP) in the absences and presence of 5-HD. ${ }^{*} P<0.05 \mathrm{vs}$. ischemia/ reoxygenation $(\mathrm{I} / \mathrm{R})$ alone. 


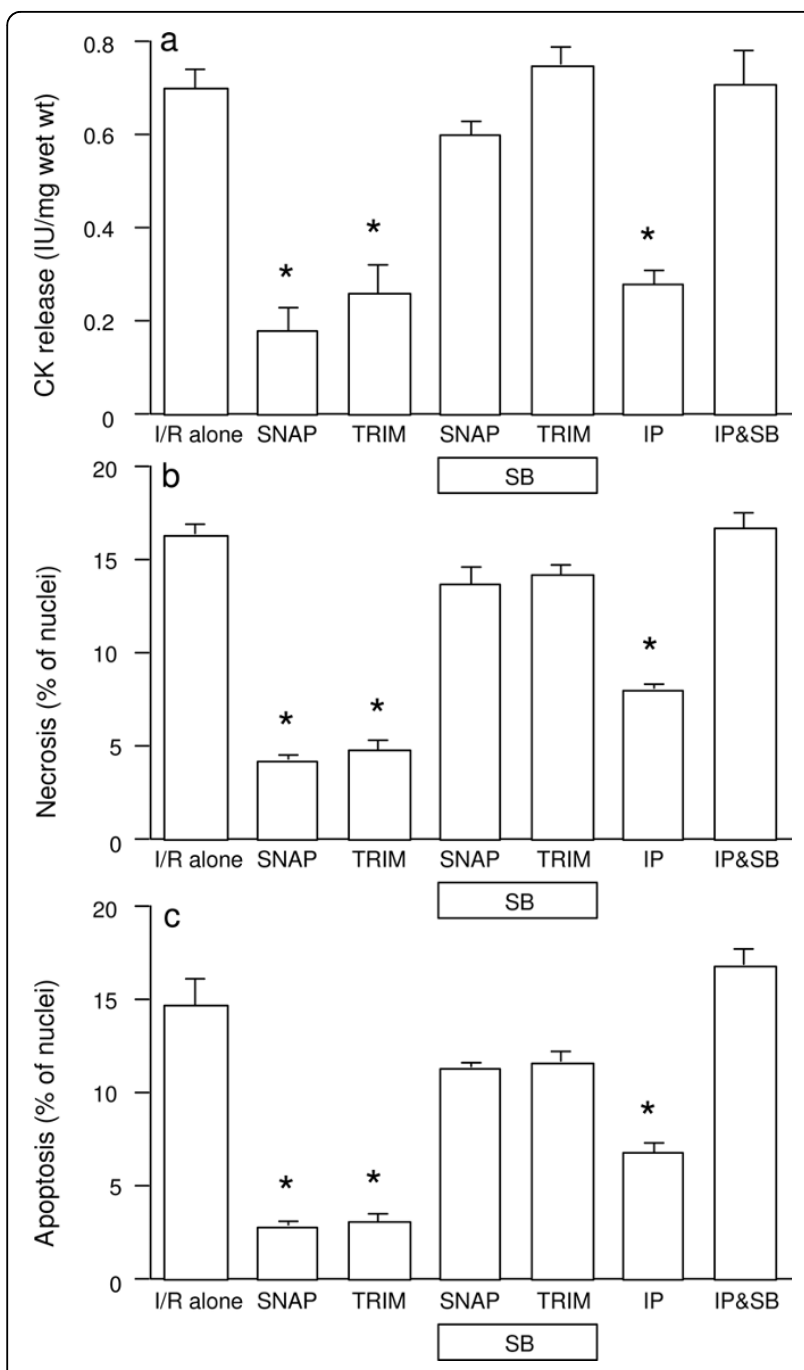

Figure 5 CK release (a), and cell necrosis (b) and apoptosis (c) in rat ventricular myocardium subjected to $90 \mathrm{~min}$ ischemia followed by $120 \mathrm{~min}$ of reoxygenation ( $n=6 /$ group). Specimens were incubated with SNAP or TRIM for 20 min prior to ischemia in the absences and presence of SB203580 (SB). For comparisons, some muscles were subjected to ischemic preconditioning (IP) in the absences and presence of SB203580. *P $<0.05$ vs. ischemia/reoxygenation (I/R) alone.

\section{Discussion and Conclusion}

The present studies have demonstrated that nNOS plays a dual role so that its presence is necessary to afford cardioprotection by IP, but at the same time its inhibition reduces myocardial ischemic/reoxygenation induced injury. In addition, they have shown that the protection obtained by the inhibition of nNOS is as potent as the exogenous administration of $\mathrm{NO}$ and that of IP, effects that are species-independent. Importantly, the action of nNOS and those of exogenous NO are exerted downstream of mitoK $K_{\text {ATP }}$ channels and upstream of p38 MAPK.
The duplicity of nNOS-derived effects seen in our studies can be explained, on one hand by a physiological production of NO that triggers the cardioprotective intracellular mechanism of interventions such as IP, and on the other hand by a possible much greater production of NO induced by ischemia/reoxygenation that is detrimental. In this connection, other investigators have also reported in an infarct size model that nNOS plays an opposite role in ischemia/reperfusion and IP [23] and that the protection of delayed IP in the rabbit heart is dependent on nNOS [12]. Since NO donors such as SNAP also have strong cardioprotective effects, it may be speculated that the amount and cell location of $\mathrm{NO}$ production would determine whether the final result is tissue protection or injury. It is also interesting to note that myocardial injury (CK release and cell death) was not increased by ischemia/reoxygenation in nNOS knockout mice, a finding supported by the absence of changes in infarct size in nNOS knockout mice $[9,11]$. By contrast, a deficit in nNOS in mice reduced the degree of cerebral ischemic injury [13], thus suggesting that the role of $\mathrm{nNOS}$ in ischemia/reoxygenation could be organ-specific.

Our studies locate the action of nNOS downstream of the mitoK $\mathrm{KTP}_{\mathrm{AT}}$ channels since the protection induced by nNOS specific inhibitor TRIM was blocked by $5-\mathrm{HD}$, a mitoK $K_{\text {ATP }}$ channel inhibitor. The finding that exogenous $\mathrm{NO}$ is also acting at the same point reinforces the view that the production of endogenous NO by nNOS is the mechanism of protection. Indeed, other laboratories have shown that the protection induced by exogenous $\mathrm{NO}$ is not affected by blockade of mitoK $\mathrm{K}_{\mathrm{ATP}}$ channels with 5HD or glibenclamide [17] and that the protection induced by the mitoK $\mathrm{K}_{\mathrm{ATP}}$ channel opener diazoxide is NO-dependent [14], further supporting the thesis that NO metabolism is a critical component of the cell survival mechanism. The different location of the two constitutive forms of NOS, nNOS and eNOS, may also suggest that NO plays a distinctive role depending on the cellular site where it is produced. This thesis gets support from the finding that the specific inhibition of nNOS was more protective than the non-specific inhibition of NOS. Certainly, this is an important question that would require further investigations.

Previously, our laboratory has shown that p38MAPK is another critical factor of IP-induced cardioprotection located beyond the mitoK $\mathrm{K}_{\mathrm{AT}}$ channels $[19,24]$. Here we have also shown that the effect of NO metabolism in the intracellular signaling pathways of cardioprotection is exerted upstream of p38MAPK, as the beneficial effects of exogenous NO donation and nNOS inhibition were abolished by the p38MAPK blocker SB203580. These findings are supported by the demonstration of protection of cardiac [20] and non-cardiac cells [22,25], 
by NO through activation of $\mathrm{p} 38 \mathrm{MAPK}$ and other MAPKs.

An additional important finding is the identical role played by nNOS in the three species studied (rat, mouse and human), suggesting that the function of the enzyme has been preserved throughout evolution. These results may have clinical implications since the findings observed from the animal studies could be extrapolated to human beings without the need for additional confirmatory investigations. Nonetheless, it is worth emphasizing that the present studies were performed in an in vitro model and therefore care must be taken when extrapolating the present results to clinical conditions. However, despite the differences between atrial and ventricular samples, the myocardium from the mouse and rat ventricles and from the human right atrial appendage, responded in a similar manner and hence the different source of myocardium should not represent a problem for the interpretation of the results. During cardiac surgery, myocardial ischemia is induced in a controlled manner and therefore it would be possible to enhance cardioprotection by manipulation of the NO metabolism. However, in the clinical setup, the heart may be subjected to stresses that can result in the expression of the inducible NOS (iNOS) and it would be important to elucidate the role of the constitutive NOS isoforms, nNOS and eNOS, in the pathophysiology of ischemia/reperfusion-induced injury under such conditions. It is worth emphasizing that, although the activity of nNOS has been shown to be increased in the late phase of delayed IP[12], to the best of our knowledge, the activity of the enzyme has not been determined in early IP. Indeed, it would be important to determine the activity of all NOS isoforms in future studies.

In conclusion, here we have shown that nNOS is a critical factor playing a dual role by participating in myocardial ischemia/reoxygenation injury, and at the same time, by promoting myocardial protection. These results are of clinical relevance for the design of therapeutic interventions aimed at reducing the myocardial damage caused by ischemia/reoxygenation.

\section{Methods}

\section{Procurement of myocardial samples}

Wister rats and C57BL/6J mice were purchased from Charles Rivers UK Ltd (Kent, UK). nNOS knockout mice (C57BL/6J) were also obtained from The Jackson Lab (Bar Harbor, Maine, USA). Animals were culled by cervical dislocation and the heart was rapidly dissected and sectioned as described below. These studies were undertaken in accordance with the guidelines on the Operation of Animals (Scientific Procedure) Act 1986. All procedures are approved by the Animal Care and Use Committee of the University of Leicester.
The right atrial appendage from patients undergoing elective heart surgery for coronary bypass surgery or aortic valve surgery was obtained prior to the initiation of cardiopulmonary bypass. Patients with atrial fibrillation, cancer, diabetes, poor LV function $(\mathrm{EF}<30 \%)$ or with additional surgical procedures or those being treated with opioids, catecholamines, or the mitoK $\mathrm{ATP}_{\mathrm{AT}}$ channel opener nicorandil were excluded from the study. The study was conducted according to Declaration of Helsinki principles and approval was obtained from the Local Ethics Committee. All participants provided informed written consent.

\section{Experimental model and preparation of myocardial samples}

The experimental preparation has been previously described and characterized [26,27]. Briefly, upon harvesting, samples (rats and mice hearts and human right atrial appendages) were immediately immersed in cold $\left(4^{\circ} \mathrm{C}\right) \mathrm{Krebs} /$ Henseleit/Hepes $(\mathrm{KHH})$ buffered medium containing (in $\mathrm{mM}$ ): $\mathrm{NaCl} 118, \mathrm{KCl} 4.8, \mathrm{NaHCO}_{3} 27.2$, $\mathrm{KH}_{2} \mathrm{PO}_{4} 1, \mathrm{MgCl}_{2} 1, \mathrm{CaCl}_{2} 1.25$, Glucose 10 and Hepes 20, pre-bubbled with $95 \% \mathrm{O}_{2} / 5 \% \mathrm{CO}_{2}$ and at a pH of 7.4. Specimens were immediately sectioned manually with a skin-graft blade (Swann-Morton Ltd, Sheffield, U.K.) to slices of 30-50 mg weight and 300-500 $\mu \mathrm{m}$ thickness. The specimens and the apparatus were kept wet during sectioning with cold and oxygenated medium. After this, specimens were equilibrated under normothermic aerobic conditions $\left(95 \% \mathrm{O}_{2} / 5 \% \mathrm{CO}_{2}\right)$ for $50-60 \mathrm{~min}$, and then subjected to $90 \mathrm{~min}$ of simulated ischemia at $37^{\circ} \mathrm{C}$, obtained by continuously bubbling the media with $95 \%$ $\mathrm{N}_{2} / 5 \% \mathrm{CO}_{2}$ in the absence of glucose and at a $\mathrm{pH}$ of 6.8 , followed by $120 \mathrm{~min}$ reoxygenation. Time matched aerobic controls were used in every experiment.

For the induction of IP, specimens were subjected to 5 min ischemia followed by 5 min reoxygenation prior to induction of the $90 \mathrm{~min}$ ischemia, a protocol that induces maximal protection in this in vitro preparation [28]. Reagents were incubated with the specimens prior to ischemia, during ischemia, or during reoxygenation in the manner and at the doses described below in the study protocols.

\section{Assessment of myocardial injury}

Myocardial injury was assessed by measurement of creatine kinase $(\mathrm{CK})$ release into the media during the 120 min reoxygenation period. The enzyme activity was measured by an ultraviolet method based on the formation of NADP employing a commercial assay kit (30-3060/R2: Abbott Laboratories, Diagnostic Division, Kent, UK) and a 96 well flat-bottom micro plate (Costar, Corning Life Sciences, Lowell, Massachusetts, USA). In this assay, NADP is reduced to NADPH and absorbance 
at $340 \mathrm{~nm}$ was measured using a spectrophotometer (Benchmark, Bio-Rad Laboratories, California, USA). Results were expressed as IU/mg wet weight.

\section{Assessment of cell death}

At the end of the experimental protocol, specimens were incubated for $15 \mathrm{~min}$ at room temperature on a rocker with $20 \mu \mathrm{g} / \mathrm{ml}$ propidium iodide (PI, SigmaAldrich, Dorset, UK) in phosphate buffered saline (PBS) at a $\mathrm{pH}$ of 7.4 , to identify the necrotic nuclei. Specimens were then washed with PBS twice for $5 \mathrm{~min}$ each time before fixation in $4 \%$ paraformaldehyde. They were kept overnight at $4-10^{\circ} \mathrm{C}$ and then transferred to sucrose $30 \%$ until the tissue sank. All the above steps were performed in the dark to avoid exposure to light. Following this, the specimens were embedded with Optical Cutting Temperature embedding matrix (Tissue-Tek ${ }^{\oplus}$, Agar Scientific Ltd, Essex, UK). Frozen sections were then cut at $7 \mu \mathrm{m}$ thickness in a Bright cryotome (model OTF) at circa $-25^{\circ} \mathrm{C}$, and sections were collected on SuperFrost Plus slides (Menzel Glasser, Braunschweig, Germany). During the preparation of slides, the periphery of specimens were discarded because this area may be more susceptible to damage by the handling. The slides were then kept at $-80^{\circ} \mathrm{C}$ until further analysis.

To assess apoptosis, the slides were brought from $-80^{\circ} \mathrm{C}$ to room temperature, washed with $20 \mathrm{mM}$ PBS, and then permeabilised for 1 minute in a microwave oven at 850 watts in $200 \mathrm{ml}$ of $0.1 \%$ Triton $\mathrm{X}-100$ in $0.1 \mathrm{M}$ Trisodium citrate buffer at a $\mathrm{pH}$ of 6.0. After this, the slides were rapidly cooled by adding $80 \mathrm{ml}$ distilled water and transferred to $20 \mathrm{mM}$ PBS solution. In addition, they were immersed into 3\% bovine serum albumin (Sigma Aldrich, Dorset, UK) in 0.1 M Tris- $\mathrm{HCl}$ buffer with $20 \%$ fetal bovine serum (Hyclone, Utah USA) at a $\mathrm{pH}$ of 7.5 for $30 \mathrm{~min}$ to block unspecific labeled activity. The terminal deoxynucleotidyl transferase (TdT) was used to incorporate fluorescein (FITC) labelled dUTP oligonucleotides to DNA strand breaks at the 3'-OH termini in a template dependent manner (TUNEL technique) for 90 min at $37^{\circ} \mathrm{C}$ in a humidity chamber using a commercially available kit (Roche Diagnostics, Penzberg, Germany).

To distinguish the total number of nuclei, sections were counter-stained with $1 \mu \mathrm{g} / \mathrm{ml} \mathrm{4}$, 6-Diamidino-2phenylindole (DAPI) (Molecular Probes, Eugene, Oregon, USA) in PBS for $1 \mathrm{~min}$. Then the slides were washed 3 times for $5 \mathrm{~min}$ each in PBS. To reduce photobleaching, the sections were mounted with antifade solution (Prolong Antifade kit, Molecular Probes, Eugene, Oregon, USA) and covered with coverslips (Menzel Glasser, Braunschweig, Germany).

A fluorescent microscope (Axiovert $200 \mathrm{M}$, Zeiss fluorescent microscope, Göttingen, Germany) at $40 \times$ magnification was used to assess necrosis and apoptosis.
At least ten fields per section, and one section per staining, were examined for each experiment. The fields were measured following the horizontal and vertical axes of the sections. PI and FITC labelled nuclei were detected by the Cy3 and EGFP channels respectively, whilst DAPI labelling was detected by the DAPI channel. Only the necrotic or apoptotic signals coinciding with DAPI were considered true events. The NIH Image software (Scion Corp, Frederick, Maryland, USA) was used to determine the total events for each field. To avoid the inclusion of artefacts, only fluorescent signals with areas greater than $16 \mu \mathrm{m}^{2}$ were counted.

\section{Study protocols}

In each study, specimens were randomly allocated to different groups ( $n=6$ /group) and specimens from one single donor were not utilized twice for the same group. Some specimens were aerobically incubated for the whole experimental time under aerobic conditions; and others, subjected to $90 \mathrm{~min}$ ischemia/120 min reoxygenation alone, served as ischemic controls. The following studies were sequentially carried out:

\section{(i) Study 1: The role of nNOS in ischemic injury}

To investigate this, specimens from ventricles of rat hearts were randomized to receive the exogenous NO donor SNAP $(100 \mu \mathrm{M})$, the non-selective NOS inhibitor L-NAME $(100 \mu \mathrm{M})$, or the selective nNOS inhibitor TRIM $(100 \mu \mathrm{M})$, for $20 \mathrm{~min}$ prior to ischemia. The selected concentrations of the agents used were found to be optimally effective in previous studies $[26,28]$.

(ii) Study 2: To elucidate whether the role of nNOS is species-dependent

This was achieved by applying a protocol identical to study 1 to specimens from mice hearts and from human right atrial appendages.

(iii) Study 3: To further characterize the role of nNOS using nNOS knockout mice

In this study, nNOS knockout mice were used to further characterize the role of nNOS following a protocol identical to the one in study 1 . IP was also applied in a group of specimens. In addition and for comparison, ischemia/reperfusion alone and IP were induced in specimens from wild type mice.

(iv) Study 4: To investigate the location of the action of exogenous NO and of nNOS inhibition in relation to mitoKATP channels and p38MAPK

For this study, myocardial slices were obtained from rat ventricles. Specimens were randomized to receive the NO donor SNAP $(100 \mu \mathrm{M})$, or the selective nNOS inhibitor TRIM $(100 \mu \mathrm{M})$, for $20 \mathrm{~min}$ prior to ischemia in the absence and presence of the mitoK ATP $_{\text {channel }}$ blocker 5-HD (1 $\mathrm{mM})$ or the p38MAPK inhibitor SB203580 $(10 \mu \mathrm{M})$ for $30 \mathrm{~min}$. In addition, some preparations were subjected to IP. 


\section{Statistical analysis}

Data are expressed as mean \pm SEM. Each reported value was obtained after subtracting the corresponding timematched aerobic control value. One way ANOVA, followed by Bonferroni's test, was used to compare the significance between groups. Analyses were performed using the SPSS program. Differences were considered to be statistically significant if $p<0.05$.

\section{Acknowledgements}

We acknowledge Spire Hospitals for a fellowship grant to Miss A Barua and also we are grateful to Mrs Nicola Harris for her assistance in the preparation of the manuscript.

\section{Author details}

${ }^{1}$ Cardiac Surgery Unit, Dept. of Cardiovascular Sciences, University of Leicester, UK. ${ }^{2}$ Dept. Cell Physiology \& Pharmacology, University of Leicester, UK. ${ }^{3}$ Department of Cardiac Surgery, Area del Cor (ACOR) and Research Institute, University Hospital Vall d'Hebron, Universitat Autònoma de Barcelona, Barcelona, Spain.

\section{Authors' contributions}

$A B$ contributed to the design of the study, carried out the laboratory work, collated and analyzed the data and drafted the manuscript. NBS and MG contributed to the design of the study, interpretation and presentation of the data and the writing of the manuscript. All authors have read and approve the final manuscript.

Received: 23 December 2009 Accepted: 13 August 2010 Published: 13 August 2010

\section{References}

1. Bolli R: Cardioprotective function of inducible nitric oxide synthase and role of nitric oxide in myocardial ischemia and preconditioning: an overview of a decade of research. J Mol Cell Cardiol 2001, 33:1897-1918.

2. Andrew PJ, Mayer B: Enzymatic function of nitric oxide synthases. Cardiovasc Res 1999, 43:521-531.

3. Takimoto Y, Aoyama T, Tanaka K, Keyamura R, Yui Y, Sasayama S: Augmented expression of neuronal nitric oxide synthase in the atria parasympathetically decreases heart rate during acute myocardial infarction in rats. Circulation 2002, 105:490-496.

4. Xu KY, Huso DL, Dawson TM, Bredt DS, Becker LC: Nitric oxide synthase in cardiac sarcoplasmic reticulum. Proc Natl Acad Sci USA 1999, 96:657-662.

5. Kanai AJ, Pearce LL, Clemens PR, Birder LA, VanBibber MM, Choi SY, et al: Identification of a neuronal nitric oxide synthase in isolated cardiac mitochondria using electrochemical detection. Proc Natl Acad Sci USA 2001, 98:14126-14131.

6. Sears CE, Bryant SM, Ashley EA, Lygate CA, Rakovic S, Wallis HL, et al: Cardiac neuronal nitric oxide synthase isoform regulates myocardial contraction and calcium handling. Circ Res 2003, 92:e52-9.

7. Jumrussirikul $P$, Dinerman J, Dawson TM, Dawson VL, Ekelund U, Georgakopoulos D, et al: Interaction between neuronal nitric oxide synthase and inhibitory $\mathrm{G}$ protein activity in heart rate regulation in conscious mice. J Clin Invest 1998, 102:1279-1285.

8. Xuan YT, Guo Y, Zhu Y, Wang OL, Rokosh G, Bolli R: Endothelial nitric oxide synthase plays an obligatory role in the late phase of ischemic preconditioning by activating the protein kinase $C$ epsilon p44/42 mitogen-activated protein kinase pSer-signal transducers and activators of transcription1/3 pathway. Circulation 2007, 116:535-544.

9. Takimoto Y, Aoyama T, Keyamura R, Shinoda E, Hattori R, Yui Y, et al: Differential expression of three types of nitric oxide synthase in both infarcted and non-infarcted left ventricles after myocardial infarction in the rat. Int J Cardiol 2000, 76:135-145.

10. Jones SP, Girod WG, Huang PL, Lefer DJ: Myocardial reperfusion injury in neuronal nitric oxide synthase deficient mice. Coron Artery Dis 2000, 11:593-597.
11. Sumeray MS, Rees DD, Yellon DM: Infarct size and nitric oxide synthase in murine myocardium. J Mol Cell Cardiol 2000, 32:35-42.

12. Wang $Y$, Kodani E, Wang J, Zhang SX, Takano H, Tang XL, et al: Cardioprotection during the final stage of the late phase of ischemic preconditioning is mediated by neuronal NO synthase in concert with cyclooxygenase-2. Circ Res 2004, 95:84-91.

13. Huang Z, Huang PL, Panahian N, Dalkara T, Fishman MC, Moskowitz MA Effects of cerebral ischemia in mice deficient in neuronal nitric oxide synthase. Science 1994, 265:1883-1885.

14. Lebuffe G, Schumacker PT, Shao ZH, Anderson T, Iwase H, Vanden Hoek TL: ROS and NO trigger early preconditioning: relationship to mitochondrial KATP channel. Am J Physiol Heart Circ Physiol 2003, 284:H299-308.

15. Sasaki N, Sato T, Ohler A, O'Rourke B, Marban E: Activation of mitochondrial ATP-dependent potassium channels by nitric oxide. Circulation 2000, 101:439-445.

16. Wang $Y$, Kudo M, Xu M, Ayub A, Ashraf M: Mitochondrial K(ATP) channel as an end effector of cardioprotection during late preconditioning: triggering role of nitric oxide. J Mol Cell Cardiol 2001, 33:2037-2046.

17. Rakhit RD, Edwards RJ, Mockridge JW, Baydoun AR, Wyatt AW, Mann GE, et al: Nitric oxide-induced cardioprotection in cultured rat ventricular myocytes. Am J Physiol Heart Circ Physiol 2000, 278:H1211-7.

18. Nakano A, Liu GS, Heusch G, Downey JM, Cohen MV: Exogenous nitric oxide can trigger a preconditioned state through a free radical mechanism, but endogenous nitric oxide is not a trigger of classical ischemic preconditioning. J Mol Cell Cardiol 2000, 32:1159-1167.

19. Loubani M, Galiñanes M: Pharmacological and ischemic preconditioning of the human myocardium: mitoK(ATP) channels are upstream and p38MAPK is downstream of PKC. BMC Physiol 2002, 2:10.

20. Kim SO, Xu Y, Katz S, Pelech S: Cyclic GMP-dependent and -independent regulation of MAP kinases by sodium nitroprusside in isolated cardiomyocytes. Biochim Biophys Acta 2000, 1496:277-284.

21. Wang D, Yu X, Brecher P: Nitric oxide and N-acetylcysteine inhibit the activation of mitogen-activated protein kinases by angiotensin II in rat cardiac fibroblasts. J Biol Chem 1998, 273:33027-33034.

22. Jun CD, Pae HO, Kwak HJ, Yoo JC, Choi BM, Oh CD, et al: Modulation of nitric oxide-induced apoptotic death of HL-60 cells by protein kinase $\mathrm{C}$ and protein kinase $A$ through mitogen-activated protein kinases and CPP32-like protease pathways. Cell Immunol 1999, 194:36-46.

23. Lu X-M, Zhang G-X, Yu Y-Q, Kimura S, Nishiyama A, Matsuyoshi $H$, et al: The opposite roles of $\mathrm{nNOS}$ in cardiac ischemia-reperfusion-induced injury and in ischemia preconditioning-induced cardioprotection in mice. J Phsyiol Sci 2009, 59:253-262.

24. Hassouna A, Matata BM, Galiñanes M: PKC-epsilon is upstream and PKCalpha is downstream of mitoKATP channels in the signal transduction pathway of ischemic preconditioning of human myocardium. Am J Physiol Cell Physiol 2004, 287:C1418-C1425.

25. Callsen D, Brune B: Role of mitogen-activated protein kinases in Snitrosoglutathione-induced macrophage apoptosis. Biochemistry 1999, 38:2279-2286.

26. Zhang JG, Galiñanes M: Role of the L-arginine/nitric oxide pathway in ischaemic/reoxygenation injury of the human myocardium. Clin Sci (Lond) 2000, 99:497-504.

27. Ghosh S, Standen NB, Galiñanes M: Preconditioning the human myocardium by simulated ischemia: studies on the early and delayed protection. Cardiovasc Res 2000, 45:339-350.

28. Handy RL, Moore PK: A comparison of the effects of L-NAME, 7-NI and L$\mathrm{NIL}$ on carrageenan-induced hindpaw oedema and NOS activity. $\mathrm{Br} J$ Pharmacol 1998, 123:1119-1126.

doi:10.1186/1472-6793-10-15

Cite this article as: Barua et al:: Dual role of nNOS in ischemic injury and preconditioning. BMC Physiology 2010 10:15. 